\section{Mussel model calls for cash to combat invading species}

Tony Reichhardt, Washington Spend now to save later - that's the message from biologists Brian Leung and David Lodge, who have made what they say is the first attempt to merge ecology and economics into a mathematical model for analysing the risks from invasive species.

Leung and Lodge, who are based at the University of Notre Dame in Indiana, used zebra mussels as a test case. These molluscs cost US industry about $\$ 100$ million a year, primarily by clogging pipes and reducing water flow to lake-side power plants.

The researchers modelled the rate at which the invaders spread and weighed up the economic factors involved, such as the costs of preventing the mussels from invading new lakes. They calculate that it is worth spending up to $\$ 324,000$ a year to stop zebra mussels from contaminating a lake with a large power plant (B. Leung et al. Proc. R. Soc. Lond. B; in the press). As the US Fish and Wildlife Service spends just $\$ \mathbf{8 2 5 , 0 0 0}$ a year controlling all non-indigenous aquatic species in the country's lakes, Leung and Lodge conclude that "a much higher value should be placed on prevention".

Environmental economics is still a relatively new field, but quantitative tools such as this may help policy-makers to decide when it pays to prevent the spread of invasive species, says Lodge. "These sorts of analyses just haven't been done," he says, even though damage caused by invasive species costs the United States about $\$ 137$ billion a year.

With no similar studies available, it is unclear how widely Leung and Lodge's results can be applied. But their model suggests that, for zebra mussels at least, the problem should not be ignored. Over five years, it showed that it was cheaper to do nothing. But for 25 years, the economic benefits of prevention were clear.

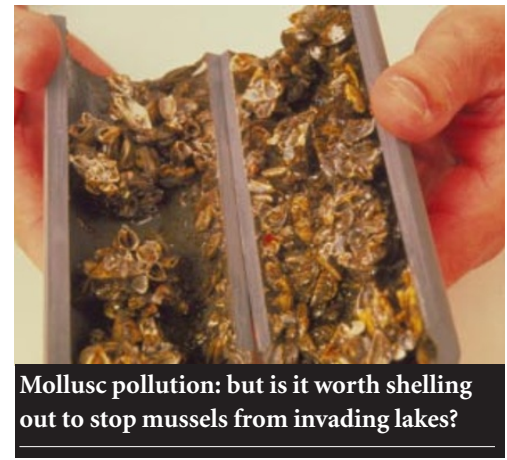

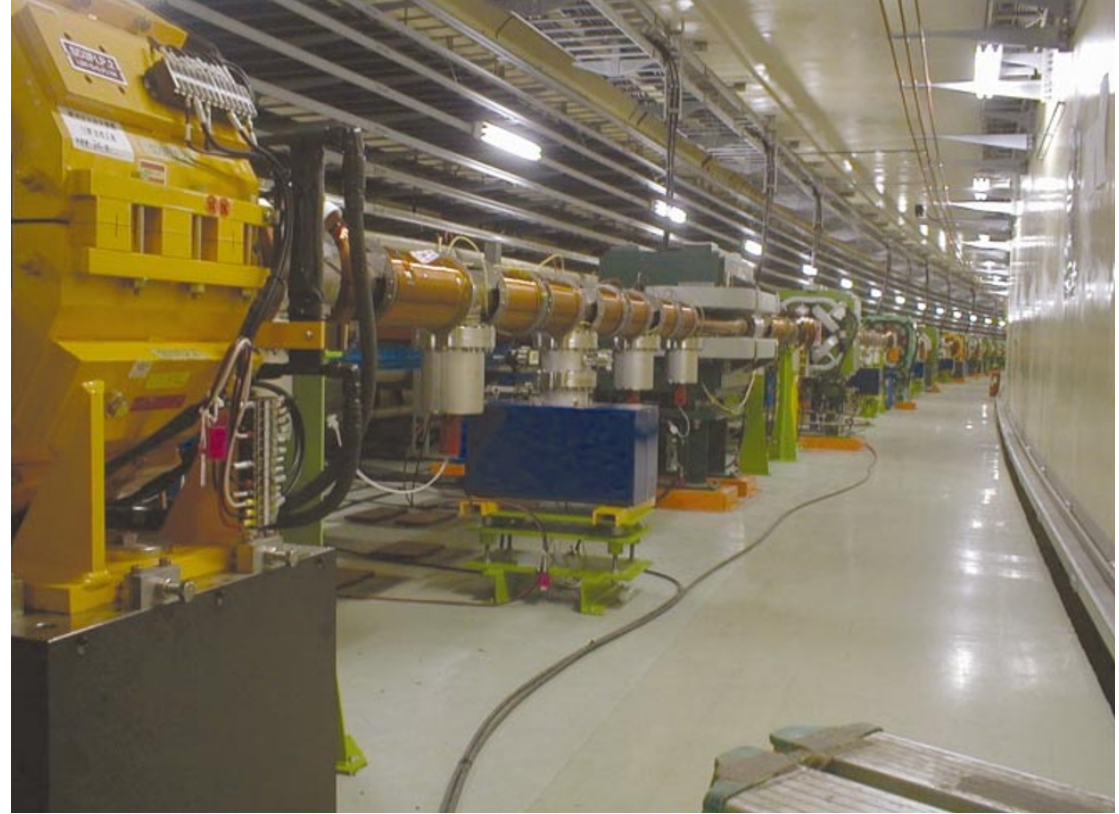

Collision course: the KEK accelerator is now producing unprecedented numbers of B mesons.
David Cyranoski, Tokyo

The cases of empty beer bottles at the High Energy Accelerator Research Organization (KEK) in Tsukuba, Japan, are testimony to the celebrations held there in recent weeks.

KEK is competing with the Stanford Linear Accelerator Center (SLAC) in the United States to produce ever higher numbers of B mesons - subatomic particles that can be used to test the standard model of high-energy physics. On 2 October, KEK surpassed the number of $\mathrm{B}$ mesons produced at SLAC, before going on to hit the 100-million mark late last month. KEK researchers say that they are now leading the race to search for physics beyond the standard model.

Both accelerators bring electrons and positrons into high-energy collisions, producing $\mathrm{B}$ mesons and their antiparticle equivalent, anti-B mesons. KEK's B-meson factory is designed to generate collisions at a higher rate than SLAC's. But technical problems left it operating at a fraction of its capacity when it started taking readings in May 1999 (see Nature 403, 586-587; 2000), allowing SLAC to generate more data.

The addition of solenoid magnets, made by winding wire around the metal that encases the beams, helped KEK to stabilize its electron and positron beams. Together with other improvements, this helped KEK to reach almost twice the rate of electron-positron collisions achieved at SLAC. The turnaround is a welcome reward for the scientists who attached the 9,000 solenoids by hand. "My hair turned grey while we were struggling with that," says KEK researcher Masakazu Yoshioka.

Both groups have already detected differences in the decay rates of the two types of B meson, which could help to explain why there is now more matter than antimatter in the Universe. Physicists believe that the two types existed in equal amounts at the Big Bang. Studying differences in the behaviour of matter and antimatter could help to explain how matter came to dominate (see Nature 418, 469; 2002).

Further data will help researchers to examine the decay rates in more detail, and could point to physics that contradicts the standard model. Kazuo Abe of the Belle collaboration at KEK, which analyses the B-meson data, says that at least 500 million, and maybe 2 billion, $B$ mesons must be produced to search for such deviations. "The high levels of production are extremely important," he says. "If KEK continues to produce data at nearly twice the rate of SLAC over the next couple of years, there will be a big difference between what we can do and what SLAC can do."

But SLAC may not lag behind for long. Its design capacity is less than that of KEK, but it is already performing above its designed collision rate. Operations at SLAC are suspended until 15 November while physicists increase the accelerator's collision rate and upgrade the detectors. "When we come back, we expect to have the same performance as Belle," says Marcello Giorgi, a physicist and spokesman for BaBar, SLAC's B-meson detector. "Perhaps in the future we will have something better."

The competition may ultimately benefit everyone involved. "KEK passes SLAC, then SLAC passes KEK - this just continues on and on," says Burt Richter, a former director of SLAC who attended the celebrations at KEK. "Having two separate groups will make sure we get honest results." 